
\title{
ES Research Square \\ Detection of Lysosomal Acid Lipase Deficiency in Tertiary Referral Centres
}

\section{See Kwok}

Manchester University https://orcid.org/0000-0002-4114-716X

Handrean Soran ( $\square$ Handrean.Soran@mft.nhs.uk)

Manchester University NHS Foundation Trust https://orcid.org/0000-0003-1714-5014

\section{Marianne Barr}

Queen Elizabeth University Hospital

\section{Research Article}

Keywords: Lysosomal acid lipase deficiency (LALD), screening, dried blood spot (DBS)

Posted Date: February 11th, 2022

DOI: https://doi.org/10.21203/rs.3.rs-1287115/v1

License: (1) This work is licensed under a Creative Commons Attribution 4.0 International License.

Read Full License 


\section{Abstract}

\section{Background}

Lysosomal acid lipase deficiency (LALD) is a rare autosomal recessive metabolic disorder. Mutations in the LIPA gene that encodes LAL result in disrupted triglyceride metabolism. Presentations and severity of the disorder are diverse and are often non-specific. Characterisations of LALD include dyslipidaemia and abnormal liver function which can overlap with conditions such as familial hypercholesterolaemia and non-alcoholic fatty liver disease (NAFLD). The rarity of LALD may mean that the condition is unrecognised or misdiagnosed. The development of effective enzyme replacement therapy for LALD has provided motivation for identifying the condition clinically. Diagnosis of LALD by genetic testing is costly. However the relatively inexpensive assay of LAL activity in dried blood spot (DBS) has made the diagnosis of LALD in clinical practice more available. We studied LALD screening in 3 cohorts: patients from hospital Lipid and Diabetes Clinics selected using the current recommended criteria, ${ }^{1}$ patients from hospital Liver Clinic who either had histological features in their liver biopsies that were consistent with LALD or who had diagnoses of non-alcoholic steatohepatitis (NASH), NAFLD or cryptogenic cirrhosis, and genetic testing for patients found to have reduced LAL activity to detect LALD carriers.

\section{Method}

Eligible patients were consented for LALD screening. A single sample of blood was obtained either from a finger prick or from a venous blood sample. The blood was transferred to a DBS filter paper for processing. LAL activity $<0.03 \mathrm{nmol} /$ punch/h was diagnostic of LALD.

Patients with reduced LAL activity were offered genetic testing. Consenting patients gave a venous blood sample of $3 \mathrm{ml}$.

\section{Results}

1730 patients were screened for LALD from 8 centres in the UK. None of the patients in the cohort tested positive for LALD. 27 patients consented to have genetic testing. No abnormalities were detected. 100 patients in the Liver cohort were screened for LALD and none was positive for LALD.

\section{Conclusion}

Using the eligibility criteria we adopted, the detection rate of LALD was meagre. In our cohorts, we did not identify any patient with undiagnosed LALD in hospital Lipid, Diabetes or Liver clinics.

\section{Introduction}

Lysosomal acid lipase deficiency (LALD) is a rare metabolic disorder resulting from mutations in the LIPA gene that encodes LAL, a key enzyme in triglyceride metabolism. The disorder is inherited in an autosomal recessive fashion and presents with two distinct phenotypes historically known as Wolman's 
disease and cholesteryl ester storage disease (CESD). Presentations of the condition are diverse and disease severity depends on the nature of the mutation and its effect on LAL function. ${ }^{1}$ Wolman's disease is the most severe form of LALD and presents in infancy with liver failure from cholesteryl ester and triglyceride accumulation. These patients rarely survive beyond 6 months of age. Patients who present later in life with CESD tend to have varying degrees of residual LAL activity and thus less severe forms of the disease. LALD is characterised by dyslipidaemia including hypertriglyceridaemia, low high-density lipoprotein cholesterol (HDLC), liver steatosis and cirrhosis. Liver biopsies of patients confirmed with LALD on genetic testing typically show microvesicular steatosis with characteristic hypertrophic Kupffer cells and portal macrophages and the presence of luminal cathepsin D and membrane markers of lysosomal-associated membrane protein (LAMP1 and LAMP2) and lysosomal integral membrane protein 2 around the lipid vacuoles. ${ }^{1,2}$ The estimated prevalence of LALD varies widely from 1 in 300,000 to 1 in $40,000 .{ }^{3}$ The non-specific presentations and diverse phenotypes of LALD overlap with other much more common conditions such as familial hypercholesterolaemia and non-alcoholic fatty liver disease (NAFLD). This, coupled with a low index of suspicion among medical professionals, means the condition has the potential to be unrecognised or misdiagnosed. ${ }^{4}$ An effective treatment for LALD is enzyme replacement therapy (ERT). ${ }^{5}$ The introduction of Sebelipase alfa as ERT has provided the incentive in diagnosing LALD in clinical practice. LALD can be confirmed by genetic testing but the procedure is costly. The development of an assay of LAL activity in dried blood spot (DBS) using Lalistat 2 as specific LAL inhibitor has very much facilitated screening for LALD. ${ }^{6}$ In this study we evaluated screening for LALD in patients attending hospital Lipid and Diabetes Clinics using criteria proposed by Reiner et al. ${ }^{1}$ We also looked at the feasibility of performing genetic testing in patients with reduced LAL activity to detect LALD carriers. And finally we assessed LAL activity in patients attending Liver Clinics who had features in their liver biopsy that might be compatible with LALD; we invited patients who had been diagnosed with non-alcoholic steatohepatitis (NASH), NAFLD or cryptogenic cirrhosis to have DBS testing for LALD.

\section{Method}

Approvals for the study were obtained from Greater Manchester Central Research Ethics Committee and Health Research Authority. The study was sponsored by Manchester University NHS Foundation Trust.

There were 3 patient cohorts in this study.

\section{Screening cohort:}

The inclusion criteria in our study were adapted from those proposed by Reiner et al. ${ }^{1}$ Eligibility included non-obese patients (BMI $\leq 30)$ who have at least 3 of the following: 1. Low HDL $(<1.0 \mathrm{mmol} / /$ in men and $<1.3 \mathrm{mmol} / \mathrm{l}$ in women). 2. Elevated triglycerides $>1.7 \mathrm{mmol} / \mathrm{l}$. 3. Elevated total cholesterol $>6.2$ or lowdensity lipoprotein cholesterol (LDLC) $>4.7 \mathrm{mmol} / \mathrm{l}$. 4. Raised liver alanine aminotransferase (ALT) $1.5 \mathrm{x}$ upper limit of normal with no known metabolic cause or viral disease or alcohol excess. 5. Diagnosed with NAFLD with or without hyperlipidaemia. 
Patients eligible for LALD screening were recruited between 2014 and 2017 from hospital Lipid and Diabetic clinics in Manchester and 7 other remote sites and consented to give a DBS sample for LAL enzyme testing. Remote sites contributing samples for LALD screening included Lipid and Diabetic Clinics from Manchester, Chorley, Devon/Exeter, Dudley, Isle of Wight, Oldham, Sandwell and Yeovil. There was no upper limit to the number of patients recruited in the study period.

The DBS samples were processed at all the recruiting sites where they were collected, batched and forwarded to the Inherited Metabolic Disease Laboratory, Royal Hospital for Children, in Glasgow. LAL enzyme activities were measured fluorimetically using 4-methylumbelliferone labelled substrate and the inhibitor Lalistat $2 .{ }^{6} \mathrm{LAL}$ activity $<0.03 \mathrm{nmol} / \mathrm{punch} / \mathrm{h}$ was accepted to be diagnostic of LALD. $\beta$ galactosidase ( $\beta$-gal) activity was measured on the study samples as a control enzyme to ascertain sample quality. Samples with $\beta$-gal activity within the normal reference range $(85-450 \mathrm{pmol} / \mathrm{punch} / \mathrm{h})$ were deemed to be of reasonable quality. All the enzyme activity results were sent from Glasgow to Manchester for collation.

II. Genetic testing to detect LALD carrier:

A previous series showed that LALD carriers had enzyme activities of $0.13-0.40 \mathrm{nmol} /$ punch $/ \mathrm{h}{ }^{6}$ Patients from the LALD screening cohort from all participating sites who had LAL activity of $<0.25$ $\mathrm{nmol} / \mathrm{punch} / \mathrm{h}$ and $\beta$-gal activity $>85 \mathrm{pmol} / \mathrm{punch} / \mathrm{h}$ were consented and invited to give a blood sample of $3 \mathrm{ml}$ for genetic testing. Blood samples from remote sites were sent to Manchester on the day of collection and on arrival in Manchester, were stored at $-80^{\circ} \mathrm{C}$. Frozen samples were batched and forwarded to Viapath, Guy's Hospital London for LALD genetic testing.

III. Liver cohort:

Patients were selected from Lipid and Liver Clinics in Manchester if they had had a liver biopsy which showed features consistent with LALD or if they had not had liver biopsy but had been diagnosed with NASH, NAFLD or cryptogenic cirrhosis. Exclusion criteria for this cohort include 1. Liver disease caused by alcohol excess, chronic viral hepatitis, haemachromatosis, primary biliary cirrhosis, primary sclerosing cholangitis, autoimmune hepatitis, Wilson's disease, alpha 1-antittrypsin deficiency. 2. HIV positive. 3. Cystic fibrosis. 4. Galactosaemia. 5. Glycogen storage disease. 6. Liver damage caused by hepatotoxic drugs or toxins.

Eligible patients who consented to DBS sampling until the proposed sample size of 100 was reached. DBS samples were forwarded to Glasgow for LAL activity assays.

\section{DBS sample}

DBS cards were provided by the Glasgow Laboratory. It is imperative when preparing blood spot samples that the filter paper is completely saturated with fresh blood. It is preferable to completely fill one circle on the card rather than partially filling all 5 circles. To prevent deterioration of enzyme activity prior to 
analysis good sample preparation and handling guidelines must be followed. The cards can be spotted from either a finger prick or from a fresh venous blood sample with the latter being transferred to the card as soon as possible after collection. The cards should be left to dry for a minimum of 4 hours but preferably overnight. Once dry, the cards are placed in a sealed plastic bag with a sachet of dessicant. They can then be stored in a refrigerator at $4^{\circ} \mathrm{C}$ until being sent to the testing laboratory at ambient temperature.

\section{Results}

1730 patients were screened for LALD between 2014 and 2017 from 8 centres in the UK using the inclusion criteria described above. The numbers of recruits from each site are shown in Table 1. Enzyme activity $<0.03 \mathrm{nmol} /$ punch/ $\mathrm{h}$ is diagnostic of LALD. ${ }^{6}$ None of the patients in our cohort was found to have LALD. LAL activity results from the screening cohort are shown in Figure 1. The mean LAL activity in our screening cohort was $0.4 \mathrm{nmol} / \mathrm{punch} / \mathrm{h}$.

Using the chosen LAL activity cut-off of $<0.25 \mathrm{nmol} / \mathrm{punch} / \mathrm{h}$ in combination with a normal $\beta$-gal activity (>85 pmol/punch/h) 37 samples from 6 sites were eligible for genetic testing for LALD carrier status. Results are shown in Table 2. 27 patients consented to have genetic testing. No pathogenic mutations were identified in any of the patients tested.

Between 2011 and 2015, 412 liver biopsies were carried out in Manchester University NHS Foundation Trust. 188 of the biopsy results showed features that might be consistent with LALD. These patients' medical records were screened for eligibility based on the defined exclusion criteria. 23 were found to be eligible and 9 patients consented to participate. In addition, patients were recruited who had been diagnosed with NASH, NAFLD or cryptogenic cirrhosis. 28 eligible participants were recruited from Lipid Clinic, 61 from Liver Clinic and 2 participants were self-volunteers. In the liver cohort of 100 participants, none was found to have LALD. Figure 2 shows the LAL activity results in the liver cohort. The mean LAL activity in this cohort was $0.35 \mathrm{nmol} /$ punch/h. Barrata et al ${ }^{7}$ described reduced LAL activities observed in NAFLD patients, we did not see this pattern in our liver cohort.

\section{Discussion}

In this study, we did not identify any patients with LALD in the 1730 patients recruited from hospital Lipid and Diabetic clinics screening using the eligibility criteria described above ${ }^{1}$. This finding is similar to the outcome of a substudy in the PATHFINDER project where laboratory databases were used to identify patients with combination of low HDLC and raised liver transaminases who were then invited for DBS testing. No LALD affected cases were identified in this cohort either. ${ }^{8}$ Similar results were reported in a paediatric population. ${ }^{9}$

Strong associations between LALD and other liver pathologies such as NASH, NAFLD and cryptogenic cirrhosis are well recognised. ${ }^{4,7}$ In our liver cohort, we did not find any patients with LALD. It would seem 
that even in patients with diagnosed NASH, NAFLD and cryptogenic cirrhosis, LALD is rare.

LALD is a rare condition and LALD carrier identification is difficult. Carriers are known to have intermediate enzyme activity. Previously a cohort of 15 confirmed carriers were found to have enzyme activities in the range of $0.13-0.40 \mathrm{nmol} / \mathrm{punch} / \mathrm{h}$ (Normal reference range $0.37-2.30) .{ }^{6}$ In our study, a cut-off of $0.25 \mathrm{nmol} / \mathrm{punch} / \mathrm{h}$ was chosen for carrier testing as it was anticipated that approximately two thirds of carriers might be captured at this level. $\beta$-gal activity within the normal reference range $(>85$ $\mathrm{pmol} / \mathrm{punch} / \mathrm{h}$ ) was used to ascertain good sample quality. However, none of the subjects identified using these cut-offs were found to have mutations within the LIPA gene. We recognise that a higher cutoff of LAL activity (e.g. $0.4 \mathrm{nmol} /$ punch/h) might capture more potential carriers, however this would mean conducting genetic testing for a much larger cohort and this was necessarily constrained by funding issues. Whilst being excellent at diagnosing LALD affected cases the DBS LAL enzyme assay is not really designed to identify carriers. The issues with sample degradation make the identification of carriers particularly challenging: the quality of DBS samples from the multiple different centres was variable and difficult to control. Ideally mutation analysis would need to be performed on all subjects to determine the true carrier frequency but as mentioned previously this was not possible due to funding constraints.

There were limitations to our study. We acknowledge that to screen for a rare condition like LALD, a much larger number of participants would be required. It is thought that LALD is an under-diagnosed condition but, even using the triggers for LAL testing of combined dyslipidaemia and liver dysfunction, the detection rate remains low. Interestingly a recent epidemiological study looking at the incidence of all currently known LAL mutations has given an estimated prevalence of 1 in 177,000 therefore confirming it to be within the ultra-rare classification of diseases. ${ }^{10}$

\section{Conclusion}

Based on the eligibility criteria we adopted, ${ }^{1}$ our results do not suggest that a significant number of patients being seen in Lipid, Diabetic or Liver Clinics have undiagnosed LALD. However given the ease of diagnostic testing using a DBS and the potential availability of ERT, testing for LALD is probably still recommended in atypical cases albeit not as first line.

\section{Declarations}

\section{Acknowledgements}

We acknowledge support from Manchester Comprehensive Local Research Network.

\section{Authors' contributions}

HS conceived the study design, SK collated the results and wrote the first draft, all authors contributed to the production of the final manuscript. All authors read and approved the final manuscript. 


\section{Funding}

Financial support for the study was received from Synageva Bio Pharma Corp and Alexion Pharmaceuticals Inc.

\section{Availability of data and materials}

Study data and materials for the study are available from the corresponding author on reasonable request.

\section{Ethics and consent}

Approvals for the study were obtained from Greater Manchester Central Research Ethics Committee and Health Research Authority (REC reference 13/NW/0297). Informed consent was obtained from all group participants.

\section{Collaborators}

Dr Ali Al-Bahrani, Isle of Wight NHS Trust; Dr Ansu Basu, Sandwell and West Birmingham NHS Trust; Professor Deepak Bhatnagar, The Pennine Acute Hospitals NHS Trust; Dr Alex Bickerton, Yeovil District Hospital NHS Foundation Trust; Professor Satyan Rajbhandari, Lancashire Teaching Hospitals NHS Foundation Trust; Dr Jacqueline Smith, The Dudley Group NHS Foundation Trust; Professor Handrean Soran, Manchester University HNS Foundation Trust; Dr Bijay Vaidya, Royal Devon and Exeter NHS Foundation Trust.

\section{Consent for publication}

Participants were made aware that anonymised results would be published in peer-reviewed journals.

\section{Competing interests}

The authors declare that they have no competing interests.

\section{References}

1. Zeljko Reiner, Ornella Guardamagna, Devaki Nair, Handrean Soran, Kees Hovingh,Stefano Bertolini, Simon Jones, Marijana Coric, Sebastiano Calandra, John Hamilton,Terence Eagleton, Emilio Ros. Lysosomal acid lipase deficiency - An under-recognized cause of dyslipidaemia and liver dysfunction. Atherosclerosis. 2014 Jul;235(1):21-30.

2. Helena Hůlková, Milan Elleder. Distinctive histopathological features that support a diagnosis of cholesterol ester storage disease in liver biopsy specimens. Histopathology 2012 Jun;60(7):1107-13.

3. Marinos Pericleous, Claire Kelly, Tim Wang, Callum Livingstone, Aftab Ala. Wolman's disease and cholesteryl ester storage disorder: the phenotypic spectrum of lysosomal acid lipase deficiency. 
Lancet Gastroenterol Hepatol 2017 Sep;2(9):670-679.

4. Donna L Bernstein, Helena Hülkova, Martin G Bialer, Robert J Desnick. Cholesteryl ester storage disease: review of the findings in 135 reported patients with an underdiagnosed disease. J Hepatol 2013 Jun;58(6):1230-43.

5. James E Frampton. Sebelipase Alfa: A Review in Lysosomal Acid Lipase Deficiency. Am J Cardiovasc Drugs 2016 Dec;16(6):461-468.

6. John Hamilton, lain Jones, Rajeev Srivastava, Peter Galloway. A new method for the measurement of lysosomal acid lipase in dried blood spots using the inhibitor Lalistat 2. Clin Chim Acta 2012 Aug 16;413(15-16):1207-10.

7. Francesco Baratta, Daniele Pastori, Maria Del Ben, Licia Polimeni, Giancarlo Labbadia, Serena Di Santo, Fiorella Piemonte, Giulia Tozzi, Francesco Violi, Francesco Angelico. Reduced Lysosomal Acid Lipase Activity in Adult Patients With Non-alcoholic Fatty Liver Disease. EBioMedicine 2015 May 22;2(7):750-4.

8. Timothy M Reynolds, Clare Mewies, John Hamilton, Anthony S Wierzbicki, PATHFINDER Project Collaboration group. Identification of rare diseases by screening a population selected on the basis of routine pathology results-the PATHFINDER project: lysosomal acid lipase/cholesteryl ester storage disease substudy. J Clin Pathol 2018 Jul;71(7):608-613.

9. Laura G Draijer, Annet M Bosch, Albert Wiegman, Barbara Sjouke, Marc A Benninga, Bart G P Koot. Screening for lysosomal acid lipase deficiency: A retrospective data mining study and evaluation of screening criteria. Atherosclerosis 2018 Nov;278:174-179.

10. Anna Carter, Simon Mark Brackley, Jiali Gao, Jake Peter Mann. The global prevalence and genetic spectrum of lysosomal acid lipase deficiency: A rare condition that mimics NAFLD. J Hepatol 2019 Jan;70(1):142-150.

\section{Tables}

Table 1. Sites and numbers recruited for LALD screening. 


\begin{tabular}{|lll|}
\hline Site & Collaborator & Number of recruits \\
\hline Dudley & Jacqueline Smith & 543 \\
\hline Chorley & Satyan Rajbhandari & 418 \\
\hline Manchester & Handrean Soran & 337 \\
\hline Sandwell & Ansu Basu & 280 \\
\hline Yeovil & Alex Bickerton & 81 \\
\hline Devon/Exeter & Bijay Vaidya & 54 \\
\hline Isle of Wight & Ali Al-Bahrani & 8 \\
\hline Oldham & Deepak Bhatnagar & 9 \\
\hline
\end{tabular}

Table 2. Samples eligible for LALD genetic testing. 


\begin{tabular}{|c|c|c|c|c|}
\hline Site & Study ID & $\begin{array}{l}\text { DBS LAL activity } \\
\text { (nmol/punch/h) }\end{array}$ & $\begin{array}{c}\text { B-gal activity } \\
\text { (pmol/punch/h) }\end{array}$ & $\begin{array}{l}\text { Genetic testing } \\
\text { outcome }\end{array}$ \\
\hline \multirow[t]{4}{*}{ Manchester } & M0115 & 0.22 & 97 & Negative \\
\hline & M0181 & 0.24 & 121 & Negative \\
\hline & M0172 & 0.22 & 348 & Negative \\
\hline & M0166 & 0.24 & 86 & Negative \\
\hline \multirow[t]{13}{*}{ Chorley } & CH363 & 0.14 & 144 & Negative \\
\hline & CH357 & 0.21 & 99 & Negative \\
\hline & $\mathrm{CH} 031$ & 0.23 & 103 & Declined \\
\hline & $\mathrm{CH} 362$ & 0.23 & 122 & Declined \\
\hline & CH356 & 0.24 & 119 & Deceased \\
\hline & CH368 & 0.24 & 120 & Negative \\
\hline & $\mathrm{CH} 216$ & 0.16 & 134 & Declined \\
\hline & $\mathrm{CH} 223$ & 0.23 & 127 & Deceased \\
\hline & $\mathrm{CH} 416$ & 0.14 & 90 & Negative \\
\hline & CH141 & 0.2 & 102 & Negative \\
\hline & CH124 & 0.2 & 115 & Declined \\
\hline & CH009 & 0.24 & 87 & Negative \\
\hline & CH099 & 0.24 & 87 & Negative \\
\hline \multirow[t]{5}{*}{ Devon } & DEV030 & 0.17 & 87 & Declined \\
\hline & DEV024 & 0.2 & 103 & Negative \\
\hline & DEV018 & 0.22 & 107 & Negative \\
\hline & DEV019 & 0.22 & 111 & Negative \\
\hline & DEV005 & 0.24 & 126 & Negative \\
\hline \multirow[t]{7}{*}{ Dudley } & DU291 & 0.19 & 135 & Negative \\
\hline & DU352 & 0.22 & 98 & Negative \\
\hline & DU097 & 0.24 & 94 & Negative \\
\hline & DU142 & 0.24 & 139 & Negative \\
\hline & DU064 & 0.23 & 195 & Negative \\
\hline & DU120 & 0.23 & 107 & Negative \\
\hline & DU160 & 0.21 & 153 & Negative \\
\hline \multirow[t]{5}{*}{ Sandwell } & SA166 & 0.22 & 99 & Declined \\
\hline & SA066 & 0.21 & 95 & Negative \\
\hline & SA091 & 0.21 & 120 & Declined \\
\hline & SA089 & 0.23 & 120 & Negative \\
\hline & SA126 & 0.14 & 200 & Declined \\
\hline \multirow[t]{3}{*}{ Yeovil } & YE072 & 0.22 & 87 & Negative \\
\hline & YE054 & 0.24 & 97 & Negative \\
\hline & YE009 & 0.2 & 104 & Negative \\
\hline
\end{tabular}

Figures 


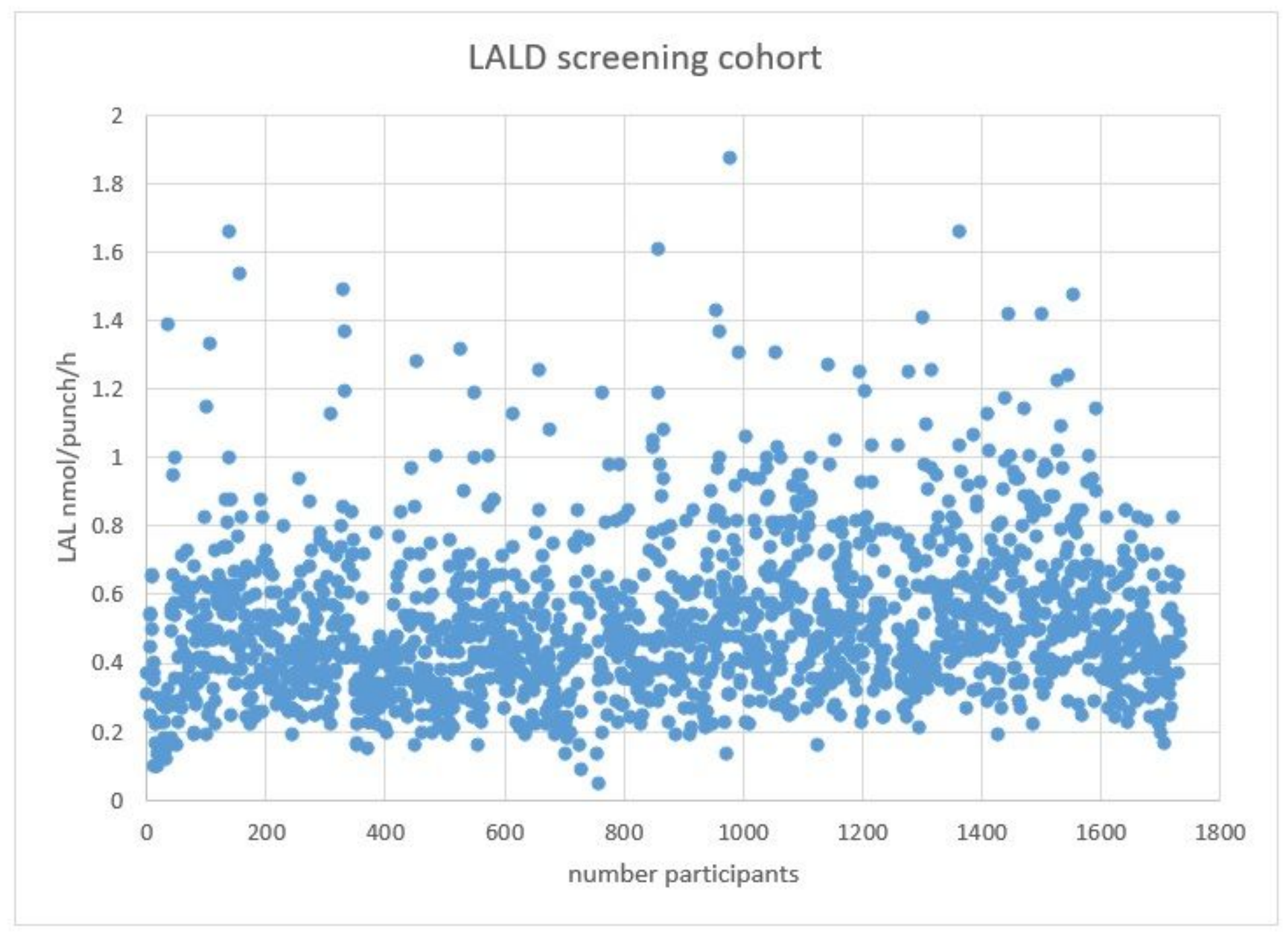

\section{Figure 1}

LAL activities in screened cohort. Mean LAL activity=0.4 nmol/punch $/ \mathrm{h}$. 


\section{liver cohort}

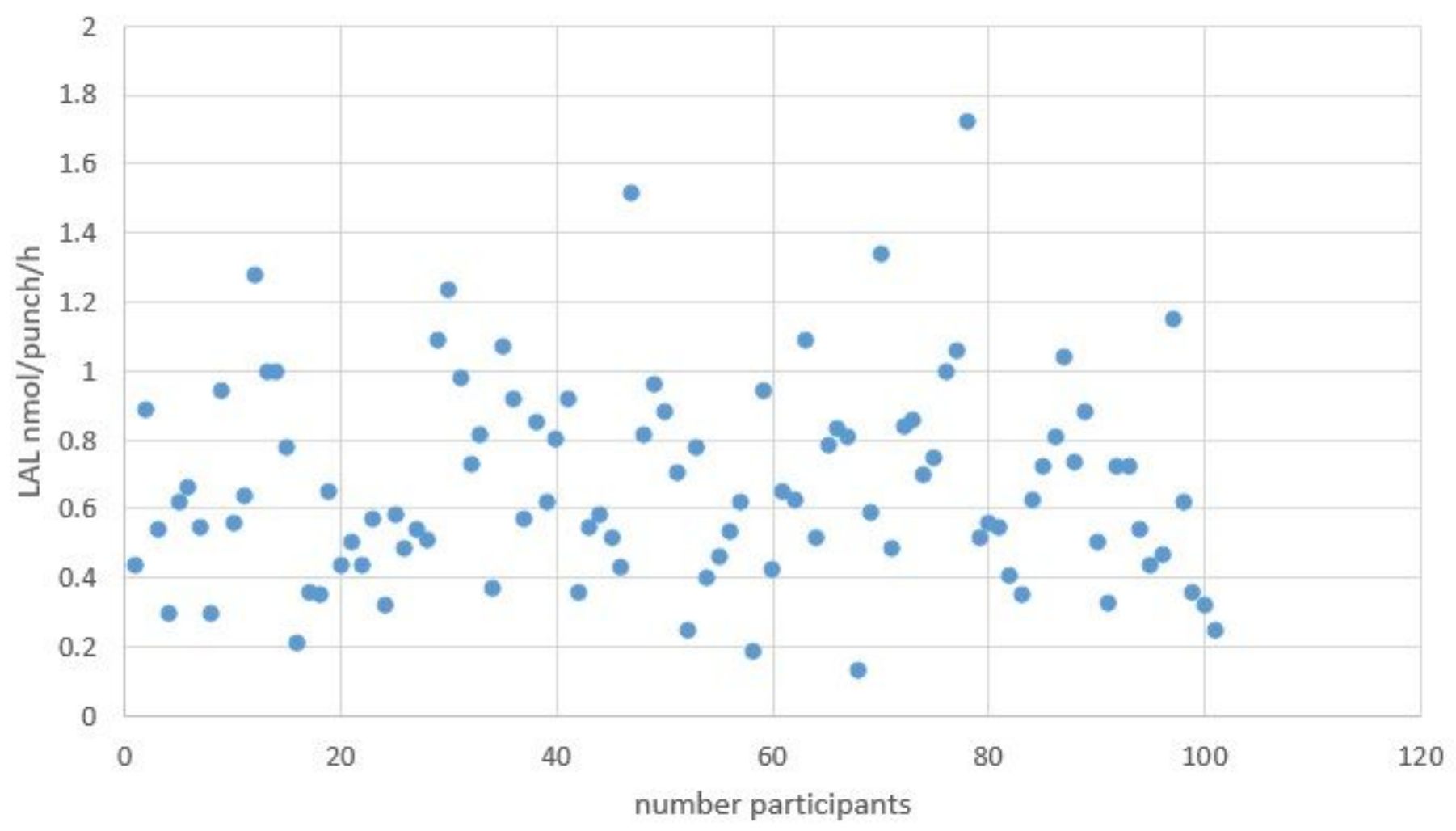

Figure 2

LAL activities in liver cohort. Mean LAL activity $=0.35 \mathrm{nmol} / \mathrm{punch} / \mathrm{h}$. 